Homology, Homotopy and Applications, vol. 8(2), 2006, pp.75-90

\title{
STABLE SPLITTINGS OF CLASSIFYING SPACES OF FINITE GROUPS: BRIDGING THE WORK OF BENSON-FESHBACH AND MARTINO-PRIDDY
}

\author{
JILL DIETZ
}

(communicated by N.J. Kuhn)

\begin{abstract}
We provide a direct connection between the work of David Benson and Mark Feshbach [2], and the work of John Martino and Stewart Priddy [9] on stable splittings of classifying spaces of finite groups.
\end{abstract}

\section{Introduction}

The journal Topology published two papers in 1992 on stable splittings of classifying spaces of finite groups. One was written by the pair of David Benson and Mark Feshbach [2], and the other by John Martino and Stewart Priddy [9]. The two papers address exactly the same question and essentially reach the same conclusion: that the $p$-complete stable splitting of a classifying space of a finite group, $B G$, is obtained from information about the contribution of summands from classifying spaces of subgroups of $G$, which in turn is obtained by studying certain simple modules. However, the approaches taken by the two pairs of authors are quite different.

Stable decompositions of $B G_{+}$can be obtained via idempotent decompositions of the identity in the ring of stable self maps, $\left\{B G_{+}, B G_{+}\right\}$. In general, if $R$ is a ring and $1=e_{1}+e_{2}+\cdots+e_{n}$ is a primitive orthogonal idempotent decomposition in $R$, then each $e_{i} R$ is an indecomposable $R$-module. If $J(R)$ is the Jacobson radical of $R$, then each $e_{i} R / e_{i} J(R)$ is a simple $R$-module. The number of copies of a particular indecomposable module $e_{i} R$ in a decomposition of $R$ is equal to the dimension of the corresponding simple module over its endomorphism ring. Neither BensonFeshbach nor Martino-Priddy directly searches for simple $\left\{B G_{+}, B G_{+}\right\}$-modules. Rather, each pair analyzes an associated ring and its simple modules.

Studying the ring $\left\{B G_{+}, B G_{+}\right\}$was made more feasible with Carlsson's [3] solution of the Segal Conjecture. Using this solution, Lewis, May, and McClure [8] showed that $\left\{B G_{+}, B G_{+}\right\}$is ring isomorphic to the completion of a kind of double Burnside ring. That is, $\left\{B G_{+}, B G_{+}\right\} \cong A(G, G)_{I}$, where completion is with respect to filtration by a certain ideal $I$.

In [2], Benson and Feshbach show that when $G=P$ is a $p$-group, indecomposable summands of $B P_{+}$correspond to simple $\bar{A}(P, P)$-modules, where $\bar{A}(P, P)=$

Received January 26, 2006, revised June 23, 2006; published on July 17, 2006. 2000 Mathematics Subject Classification: 55R35.

Key words and phrases: Classifying spaces of groups, stable homotopy theory.

Copyright (C) 2006, International Press. Permission to copy for private use granted. 
$\mathbb{F}_{p} \otimes_{\mathbb{Z}} A(P, P)$. They define a "coadjoint" module for $\bar{A}(P, P)$ whose subquotients are either zero or simple. The simple modules are parametrized by "types" of subgroups $Q \leqslant P$ and by simple $\mathbb{F}_{p}$ Out $Q$-modules, and form a set of representatives for the homotopy types of indecomposable summands of $B P_{+}$. Again, the multiplicity of a summand in $B P_{+}$is equal to the dimension of the corresponding simple $\bar{A}(P, P)$-module over its endomorphism ring. Further details on the BensonFeshbach method are given in Section 3.

In [9], Martino and Priddy use Nishida's [10] application of the Segal Conjecture showing that for all finite $p$-groups $P, \mathbb{Z}_{p}$ Out $P \cong\{B P, B P\} / J(P)$, where $J(P)$ is the ideal of $\{B P, B P\}$ generated by all maps of the form $B P \rightarrow B K \rightarrow B P$, with $K \lesseqgtr P$. They obtain splittings of $B P$ from information on simple $\mathbb{F}_{p}$ Out $Q$ modules, $Q \leqslant P$. The multiplicity of a summand $X$ in $B P$, denoted $m(X, B P)$, is determined by measuring the degree of linear independence among the contributions to $m(X, B P)$ from classifying spaces of subgroups of $P$. Further details on the Martino-Priddy method are given in Section 4.

In his reviews of the two papers, John Harris [7] wrote: "It would be interesting to see a direct proof that these two [computations of $m(X, B G)$ ] are the same." Similarly, in Benson's [1] survey of developments in the study of stable splittings of classifying spaces of finite groups, he asked "What is the precise relationship between the matrices of Martino and Priddy and the modules of Benson and Feshbach?" This paper answers Harris and Benson's questions.

The organization of this paper is as follows: In Section 2, we remind the reader of the basic theory of stable splittings. This basic theory is the launching point for the two papers [2] and [9]. Sections 3 and 4 give further details on the Benson-Feshbach and Martino-Priddy methods respectively. Section 5 provides a direct connection between the two theories. We give an example in Section 6 which shows how to compute the multiplicity of a summand in the splitting of a certain $B P$ using each method, and illustrate the connection between the two methods.

\section{Stable Splittings}

Stably, we have

$$
B G_{+} \simeq \bigvee_{p|| G \mid}\left(B G_{+}\right)_{p}^{\hat{p}}
$$

where $\left(B G_{+}\right)_{p}$ denotes the $p$-completion of $B G_{+}$. It makes sense, then, to fix a prime $p$ and study the $p$-local stable decomposition of $B G_{+}$. Moreover, $\left(B G_{+}\right)_{p}$ appears as a summand of $B P_{+}$, where $P$ is a $p$-Sylow subgroup of $G$. Thus, we will concentrate on the case when $G=P$ is a $p$-group. Throughout this paper $p$ will be a prime, $P$ a $p$-group, $B P_{+}$the $p$-completion of the suspension spectrum of the classifying space with disjoint basepoint, and $\left\{B P_{+}, B P_{+}\right\}$the ring of $p$-complete self maps. We will always work in the category of $p$-complete spectra.

A decomposition $B P_{+} \simeq X_{1} \vee X_{2} \vee \cdots \vee X_{n}$ corresponds to an idempotent decomposition in $\left\{B P_{+}, B P_{+}\right\}, 1=e_{1}+e_{2}+\cdots+e_{n}$. A summand $X_{i}=e_{i} B P_{+}$ is the mapping telescope $\operatorname{Tel}\left(B P_{+} \stackrel{e_{i}}{\rightarrow} B P_{+} \stackrel{e_{i}}{\rightarrow} \cdots\right)$. 
The Segal Conjecture relates the ring $\left\{B P_{+}, B P_{+}\right\}$to a double Burnside ring $A(P, P)$ as follows. Let $G$ and $G^{\prime}$ be two finite groups. $A\left(G, G^{\prime}\right)$ is the Grothendieck group of isomorphism classes of finite $G \times G^{\prime}$-sets with free right $G^{\prime}$ action. Given $H \leqslant G$ and a homomorphism $\phi: H \rightarrow G^{\prime}$, let $X_{H, \phi}=\left(G \times G^{\prime}\right) / \triangle_{H, \phi}$, where $\triangle_{H, \phi}=\{(h, \phi(h)) \mid h \in H\}$. Every transitive $G^{\prime}$-free $G \times G^{\prime}$-set is of this form, so $A\left(G, G^{\prime}\right)$ is free abelian with basis elements $\zeta_{H, \phi}$ corresponding to conjugacy classes of pairs $(H, \phi)$.

Multiplication, $A\left(G, G^{\prime}\right) \times A\left(G^{\prime \prime}, G\right) \rightarrow A\left(G^{\prime \prime}, G^{\prime}\right)$, is given by a double coset formula (see [2], Formula 2.1). When $G=G^{\prime}=G^{\prime \prime}$, we get a ring structure on $A(G, G)$.

There is a homomorphism $\alpha: A\left(G, G^{\prime}\right) \rightarrow\left\{B G_{+}, B G_{+}^{\prime}\right\}$ sending $\zeta_{H, \phi}$ to the composite

$$
B G_{+} \stackrel{t r_{+}}{\longrightarrow} B H_{+} \stackrel{B \phi_{+}}{\longrightarrow} B G_{+}^{\prime},
$$

where $t r_{+}$is the unreduced transfer map. The map $\alpha$ is an isomorphism only after completion with respect to a filtration given by powers of a certain ideal $I$ :

$$
A\left(G, G^{\prime}\right)_{I} \cong\left\{B G_{+}, B G_{+}^{\prime}\right\} .
$$

It is convenient to eliminate the disjoint basepoint. Since $B G_{+} \simeq S^{0} \vee B G$, splitting $B G_{+}$and splitting $B G$ are equivalent problems.

There is an augmentation homomorphism $\varepsilon: A\left(G, G^{\prime}\right) \rightarrow A(G, 1)$. Let $\tilde{A}\left(G, G^{\prime}\right)=$ Ker $\varepsilon$. Then we have an isomorphism

$$
\tilde{A}\left(G, G^{\prime}\right)_{I} \cong\left\{B G, B G^{\prime}\right\} .
$$

When $P$ is a $p$-group, $I$-adic completion on $\tilde{A}(P, P)$ is the same as $p$-adic completion so we have

$$
\tilde{A}(P, P)_{p}:=\mathbb{Z}_{p} \otimes \tilde{A}(P, P) \cong \tilde{A}\left(P, P \hat{)_{I}} \cong\{B P, B P\} .\right.
$$

Further reducing $\mathbb{Z}_{p}$ to its residue field $\mathbb{F}_{p}$ and letting $\tilde{A}_{p}(P, P)=\mathbb{F}_{p} \otimes_{\mathbb{Z}} \tilde{A}(P, P)_{p}$, we get maps

$$
\{B P, B P\} \stackrel{\cong}{\rightrightarrows} \tilde{A}(P, P)_{p} \rightarrow \tilde{A}_{p}(P, P) .
$$

From the idempotent refinement theorem, we see that a primitive orthogonal idempotent decomposition of the identity in $\tilde{A}_{p}(P, P)$ lifts to one in $\tilde{A}(P, P)_{p}$. Thus, there is a one-to-one correspondence between stable homotopy types of indecomposable summands of $B P$ and isomorphism types of simple $\tilde{A}_{p}(P, P)$-modules.

As mentioned in Section 1, Nishida [10] used the Segal Conjecture to show that for all finite $p$-groups $P$, the composite

$$
\mathbb{Z}_{p}^{\wedge} \text { Out } P \rightarrow\{B P, B P\} \rightarrow\{B P, B P\} / J(P)
$$

is an isomorphism of rings.

Definition 2.1. If a primitive idempotent $e \in\{B P, B P\}$ is not in $J(P)$, then the summand $e B P$ is said to originate in $B P$. Every indecomposable summand of $B P$ originates in some $B Q, Q \leqslant P$ (see [10], where the term "dominant" is used instead of "originate"). 
If $X=e B Q$ originates in $B Q$, then there is a corresponding idempotent $\hat{e} \in$ $\mathbb{Z}_{p}$ Out $Q$. Reducing $\bmod p$, we get an idempotent $\bar{e} \in \mathbb{F}_{p}$ Out $Q$. Though it is not necessarily primitive, we do obtain a simple right $\mathbb{F}_{p}$ Out $Q$-module $M=\bar{e} R / \bar{e} J(R)$, where $R=\mathbb{F}_{p}$ Out $Q$. The relationship $X \leftrightarrow M$ is a one-to-one correspondence between homotopy classes of original indecomposable summands of $B Q$ and isomorphism classes of simple right $\mathbb{F}_{p}$ Out $Q$-modules (see [11], Proposition 1.2).

A truly seminal theorem in the field of stable splittings came from Priddy in 1988. Before we restate his result, we need some notation. If $R=\mathbb{F}_{p} G$ is a group ring and $M$ is a right $R$-module, let $M^{*}$ denote the corresponding left $R$-module with action given by $r \cdot m^{*}=m \cdot r^{*}$, where $r^{*}$ denotes the image of $r \in R$ under the anti-automorphism $\sum a_{g} g \mapsto \sum a_{g} g^{-1}$. Other notation: the conjugation homomorphism is $c_{g}(a)=g a g^{-1},{ }^{g} K=g K g^{-1}$, and for $H, K \leqslant P$ we define $N(H, K)=$ $\left\{x \in P \mid H \leqslant{ }^{x} K\right\}$.

Theorem 2.2. ([11], Theorem 0.1) Let $X$ be an original summand of $B Q, Q \leqslant P$. Then $X$ is a summand of $B P$ if and only if there exist subgroups $Q^{\prime} \leqslant P^{\prime} \leqslant P$, $Q^{\prime} \cong Q$, and a retraction $\pi: P^{\prime} \rightarrow Q^{\prime}$, such that $\gamma \cdot M^{*} \neq 0$, where $M$ is the simple right $\mathbb{F}_{p}$ Out $Q^{\prime}$-module corresponding to $X$, and

$$
\gamma=\sum_{x} Q^{\prime} \stackrel{c_{x}-1}{\rightarrow} x^{-1} Q^{\prime} x \leqslant P^{\prime} \stackrel{\pi}{\rightarrow} Q^{\prime}
$$

with the sum running over coset representatives of $N\left(Q^{\prime}, P^{\prime}\right) / P^{\prime}$ such that the composition $\pi \circ c_{x^{-1}}$ is in Out $Q^{\prime}$.

This same theorem appears in [2] (as Theorem 5.2) with slightly different notation and a different proof. There the sum above runs over double coset representatives $x$ of $Q^{\prime}$ and $P^{\prime}$ in $P$, satisfying the additional condition that $Q^{\prime} \leqslant{ }^{x} P^{\prime}$.

Catalano explains that the Benson-Feshbach conditions can be replaced by $x \in$ $P / P^{\prime}$ and $Q^{\prime} \leqslant{ }^{x} P^{\prime}$, that, in turn, are equivalent to $x \in N\left(Q^{\prime}, P^{\prime}\right) / P^{\prime}$ (see remarks in [4] subsequent to Lemma 2.1 and Theorem 2.3).

Definition 2.3. Any subgroup $Q^{\prime}$ of $P$ satisfying $\gamma \cdot M^{*} \neq 0$ as above is called a contributor, and $Q^{\prime}$ contributes to the multiplicity of $X$ in $B P$. If a summand $X$ of $B K$ appears in the splitting of $B P$, then $K$ is isomorphic to a contributor (see [4], p. 35).

\section{The Benson-Feshbach Method}

Benson-Feshbach define a coadjoint module $M(P, P)$ over $A(P, P)$ as follows. Let $Q \leqslant P, \psi: Q \rightarrow P^{\prime}$, and $X=X_{Q, \psi}$, and define

$$
\begin{aligned}
f_{Q, \psi}: A\left(P, P^{\prime}\right) & \rightarrow \mathbb{Z} \quad \text { by } \\
X & \mapsto\left|X^{\triangle_{Q}, \psi}\right|
\end{aligned}
$$

Define $M\left(P, P^{\prime}\right)$ to be the free abelian group with one basis element $f_{Q, \psi}$ for each conjugacy class of pairs $(Q, \psi)$. There is an action

$$
A\left(P_{1}, P_{2}\right) \times M\left(P_{1}, P_{3}\right) \rightarrow M\left(P_{2}, P_{3}\right)
$$


given by

$$
\zeta_{H, \phi} \cdot f_{Q, \psi}=\sum_{\substack{x \in Q \backslash P P_{1} / H \\ Q \leqslant x \\ Q \cap^{x}(\operatorname{Ker} \phi) \leqslant \operatorname{Ker} \psi}} f_{\phi\left(x^{-1} Q\right), \psi \circ c_{x} \circ \phi^{-1}}([\mathbf{2}], \text { Prop. 3.1). }
$$

If $P_{1}=P_{2}=P_{3}=P$, then $M(P, P)$ is an $A(P, P)$-module.

Let $\bar{M}(P, P)=\mathbb{F}_{p} \otimes_{\mathbb{Z}} M(P, P)$ and $\bar{A}(P, P)=\mathbf{F}_{p} \otimes_{\mathbb{Z}} A(P, P)$. Every simple $\bar{A}(P, P)$-module is a composition factor of $\bar{M}(P, P)$ ([2], Lemma 3.4). There is an $\bar{A}(P, P)$-invariant filtration of $\bar{M}(P, P)$ given by what Benson-Feshbach call "types." The subquotients arising from the filtration will be the simple modules we want.

Definition 3.1. ([2], Definition 4.3) Let $Q_{1}, Q_{2} \leqslant P$ and let $\psi_{i}: Q_{i} \rightarrow P$ be homomorphisms. Write $\left(Q_{1}, \psi_{1}\right) \succeq\left(Q_{2}, \psi_{2}\right)$ if there is a surjective homomorphism $\alpha: Q_{1} \rightarrow Q_{2}$ which extends to a homomorphism $Q_{1} C_{P}\left(Q_{1}\right) \rightarrow P$, and there is an element $g \in P$ such that $\psi_{1}=c_{g} \circ \psi_{2} \circ \alpha$. If $\left(Q_{1}, \psi_{1}\right) \succeq\left(Q_{2}, \psi_{2}\right)$ and $\left(Q_{2}, \psi_{2}\right) \succeq$ $\left(Q_{1}, \psi_{1}\right)$, then we write $\left(Q_{1}, \psi_{1}\right) \sim\left(Q_{2}, \psi_{2}\right)$ and say that $\left(Q_{1}, \psi_{1}\right)$ has the same type as $\left(Q_{2}, \psi_{2}\right)$. If $\psi_{1}$ and $\psi_{2}$ are isomorphisms, then by abuse of notation we write $Q_{1} \sim Q_{2}$ and talk of types of subgroups.

The partial order $\succeq$ defines an $\bar{A}(P, P)$-invariant filtration of $\bar{M}\left(P, P^{\prime}\right)$ ([2], Proposition 4.4). Filtered quotients are denoted $\bar{L}\left(P, P^{\prime}\right)_{Q, \psi}$ and $\bar{L}(P, Q)=$ $\oplus \bar{L}(P, Q)_{Q, \psi}$, where the sum is taken over all types with $\psi$ an isomorphism.

Proposition 3.2. ([2], Proposition 4.8) The module $\bar{L}(P, Q)$ has a basis consisting of the $\bar{f}_{Q^{\prime}, \psi^{\prime}}$, where $Q^{\prime}$ has the same type as $Q$ and $\psi^{\prime}$ is an isomorphism from $Q^{\prime}$ to $Q$. A left action of $\mathbb{F}_{p}$ Out $Q$ on $\bar{L}(P, Q)$ is defined by $\eta \cdot \bar{f}_{Q^{\prime}, \psi^{\prime}}=\bar{f}_{Q^{\prime}, \eta \circ \psi^{\prime}}$, for $\eta \in$ Out $Q$. Moreover, if $M$ is a simple right $\mathbb{F}_{p}$ Out $Q$-module then $M \otimes_{\mathbb{F}_{p} \text { Out } Q} \bar{L}(P, Q)$ is a left $\bar{A}(P, P)$-module with action given by

$$
\zeta_{H, \phi} \cdot\left(s \otimes \bar{f}_{Q^{\prime}, \psi^{\prime}}\right)=\sum_{\substack{x \in Q^{\prime} \backslash P / H \\ Q^{\prime} \leqslant \operatorname{Stab} P M x_{H} \\ Q^{\prime} \cap x(\operatorname{Ker} \phi)=1}} s \otimes f_{\phi\left(x^{-1} Q^{\prime}\right), \psi \circ c_{x} \circ \phi^{-1}} .
$$

We write $\operatorname{Stab}_{P} M$ for the kernel of the composite

$$
N_{P}\left(Q^{\prime}\right) \rightarrow \text { Out } Q^{\prime} \stackrel{\cong}{\rightrightarrows} \text { Out } Q \rightarrow \text { Aut } M .
$$

From Michael Catalano we have a bit more information as follows. Let $Q_{i}$ be a conjugacy class representative of $Q$ in $P$ that has the same type as $Q$, and let $\overline{N_{P}\left(Q_{i}\right)}$ be the image of the map

$$
\begin{aligned}
& N_{P}\left(Q_{i}\right) \quad \rightarrow \quad \text { Out } Q_{i} \rightarrow \quad \text { Out } Q \\
& g \quad \mapsto \quad c_{g} \quad \mapsto \psi_{i} \circ c_{g} \circ \psi_{i}^{-1}
\end{aligned}
$$

(see [2], Lemma 5.3). In [4], Lemma 2.1, we see that

$$
M \otimes_{\mathbb{F}_{p} \text { Out } Q} \bar{L}(P, Q) \cong \oplus_{i=1}^{k} M \otimes_{\mathbb{F}_{p} \text { Out } Q} \bar{f}_{Q_{i}, \psi_{i}} .
$$

Furthermore, $M \otimes_{\mathbb{F}_{p} \text { Out } Q} \bar{f}_{Q_{i}, \psi_{i}} \cong M / \overline{N_{P}\left(Q_{i}\right)}$ (see [5], p. 16). We end up with the following proposition. 
Proposition 3.3. (Catalano) As $\mathbb{F}_{p}$-spaces, we have

$$
M \otimes_{\mathbb{F}_{p} \text { Out } Q} \bar{L}(P, Q) \cong \oplus_{i=1}^{k} M / \overline{N_{P}\left(Q_{i}\right)}
$$

where $k$ is the number of conjugacy classes of subgroups of $P$ of the same type as $Q, Q_{i}$ is a conjugacy class representative, and $\psi_{i}: Q_{i} \rightarrow Q$ is an isomorphism.

Benson-Feshbach define a certain maximal submodule $\mathcal{M}$ of $M \otimes_{\mathbb{F}_{p} \text { Out } Q} \bar{L}(P, Q)$ inductively ([2], Definition 4.9), but Catalano proves $\mathcal{M}=\mathcal{M}_{0}([\mathbf{4}]$, Lemma 3.1). Thus

$$
\mathcal{M}=\mathcal{M}_{0}=\cap_{\operatorname{Im} \phi \cong Q} \operatorname{Ker} \zeta_{L, \phi}
$$

Let $\bar{L}(P, Q, M)=\left(M \otimes_{\mathbb{F}_{p} \text { Out } Q} \bar{L}(P, Q)\right) / \mathcal{M}$.

Theorem 3.4. ([2], Theorem 5.7) The modules $\bar{L}(P, Q, M)$ are either simple or zero. As $Q$ runs through the types of subgroups of $P$ and $M$ runs through simple $\mathbb{F}_{p}$ Out $Q$-modules, the non-zero modules of the form $\bar{L}(P, Q, M)$ run through a complete set of representatives for the isomorphism types of simple $\bar{A}(P, P)$-modules.

The module $\bar{L}(P, Q, M)$ is zero if and only if the original summand of $B Q_{+}$ corresponding to $M$ does not appear as a summand of $B P_{+}$. Alternatively, the module $\bar{L}(P, Q, M)$ is non-zero if and only if the conditions of Theorem 2.2 hold (specifically, there must exist $Q \cong Q^{\prime} \leqslant P^{\prime}$ such that $\gamma \cdot M^{*} \neq 0$ ). Finally, if $X$ originates in $B Q$ then the multiplicity of $X$ in $B P$ equals the dimension of $\bar{L}(P, Q, M)$ as a module over its endomorphism ring, $\operatorname{End}_{\bar{A}(P, P)} \bar{L}(P, Q, M)$.

There is a ring homomorphism $\rho: \bar{A}(P, P) \rightarrow \mathbb{F}_{p}$ Out $P$ defined by $\rho\left(\zeta_{H, \phi}\right)=0$ unless $H=P$ and $\phi$ is an automorphism, in which case $\rho\left(\zeta_{H, \phi}\right)=\bar{\phi}$ where $\bar{\phi}$ is the outer automorphism corresponding to $\phi([\mathbf{2}]$, p.166). Clearly $\rho$ is a split surjection. A primitive idempotent is in Ker $\rho$ if and only if the corresponding indecomposable summand of $B P$ does not originate in $B P$.

If $X=e B P$ is an original indecomposable summand of $B P$, then there is a primitive idempotent $\tilde{e} \in \bar{A}(P, P)$ that is not in Ker $\rho$. Thus, there is a non-trivial idempotent $\bar{e} \in \mathbb{F}_{p}$ Out $P$ such that $\rho(\tilde{e})=\bar{e}$. We see that $m(X, B P)$ equals the dimension of $M=\bar{e} R / \bar{e} J(R)$ over its endomorphism ringEnd $\mathbb{F}_{p}$ Out $P M$.

\section{The Martino-Priddy Method}

Let $X$ be an original indecomposable summand of $B Q, Q \leqslant P$, with corresponding simple right $\mathbb{F}_{p}$ Out $P$-module $M$. As in [9], define Split $Q$ to be the set of conjugacy classes of retractions $\pi_{j}: P_{j} \rightarrow Q_{j}$, where $Q_{j} \cong Q$. For all $j$, fix isomorphisms $\psi_{j}: Q_{j} \rightarrow Q$.

Let

$$
\omega_{i j}=\sum_{N\left(Q_{i}, P_{j}\right) / P_{j}} \psi_{j} \circ \pi_{j} \circ c_{x^{-1}} \circ \psi_{i}^{-1}
$$

where the sum runs over coset representatives of $N\left(Q_{i}, P_{j}\right) / P_{j}$ such that the composition

$$
Q \stackrel{\psi_{i}^{-1}}{\rightarrow} Q_{i} \stackrel{c_{x-1}}{\rightarrow} x^{-1} Q_{i} x \leqslant P_{j} \stackrel{\pi_{j}}{\rightarrow} Q_{j} \stackrel{\psi_{j}}{\rightarrow} Q
$$


is in $\operatorname{Out} Q$.

Let $n=\mid$ Split $Q \mid$, then $\mathcal{A}(Q)=\left(\omega_{i j}\right)$ is in $\operatorname{Mat}_{n}(R)$, where $R=\mathbb{F}_{p}$ Out $Q$. Let $\mathcal{K}=\operatorname{End}_{R} M$. If $\operatorname{dim}_{\mathcal{K}} M^{*}=m$, then each $\omega_{i j}$ can be viewed as an $m \times m$ matrix over $\mathcal{K}$, where $\omega_{i j}$ acts on the left of the module $M^{*}$. Define $W_{i j}$ to be the matrix in $\operatorname{Mat}_{m}(\mathcal{K})$ corresponding to $\omega_{i j}$, and let $\mathcal{A}\left(Q, M^{*}\right)=\left(W_{i j}\right) \in \operatorname{Mat}_{n m}(\mathcal{K})$.

Theorem 4.1. ([9], Theorem 0.1) Let $X$ be an original summand of $B Q, Q \leqslant P$, with corresponding simple right $\mathbb{F}_{p}$ Out $Q$-module $M$. Then, the multiplicity of $X$ in $B P$ is $m(X, B P)=\operatorname{rank}_{\mathcal{K}} \mathcal{A}\left(Q, M^{*}\right)$.

Note that if $X$ is an original summand of $B P$, then Split $P=\{i d: P \rightarrow P\}$ and

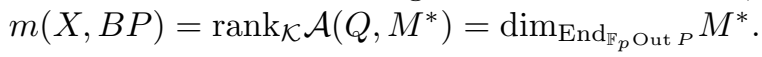

\section{Connecting Benson-Feshbach and Martino-Priddy}

We have seen that Benson-Feshbach and Martino-Priddy compute the multi-

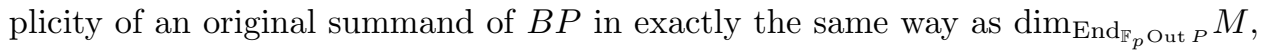
but their computations of $m(X, B P)$ for non-original summands differ vastly. This section will show a direct connection between the two computations for $m(X, B P)$ when $X$ originates in $B Q$ for some $Q \lesseqgtr P$.

\subsection{Reducing Martino-Priddy's Matrix}

If $X$ is a non-original summand of $B P$, Benson-Feshbach show there is only one type of contributor $Q$ as follows.

Lemma 5.1. (see Proposition 5.9 of [2] and Proposition 2.4 of [4]) If $Q^{\prime}$ is a contributor to $m(X, B P)$, then $Q^{\prime}$ is a direct summand of $Q^{\prime} C_{P}\left(Q^{\prime}\right)$.

The proof of this result in [2] relies almost entirely on that paper's Theorem 5.2, which has a Martino-Priddy analogue (as noted after Theorem 2.2 in this paper). Thus, we may use this Lemma in the Martino-Priddy context.

Theorem 5.2. ([2], p.158) Let $X$ be a summand of $B P$ that originates in $B Q$, $Q \lesseqgtr P$. If both $Q_{1} \cong Q$ and $Q_{2} \cong Q$ contribute to $m(X, B P)$, then $Q_{1}$ and $Q_{2}$ have the same type.

Proof. To show $\left(Q_{1}, \psi_{1}\right) \succeq\left(Q_{2}, \psi_{2}\right)$, we let $\alpha$ be the composite $Q_{1} \stackrel{\psi_{1}}{\rightarrow} Q \stackrel{\psi_{2}^{-1}}{\rightarrow} Q_{2}$. Since $Q_{1}$ is a contributor, Lemma 5.1 implies $Q_{1} C_{P}\left(Q_{1}\right)=Q_{1} \times A_{1}$ for some $A_{1} \leqslant$ $P$. We see that $\alpha$ extends to $Q_{1} C_{P}\left(Q_{1}\right)$ via

$$
Q_{1} C_{P}\left(Q_{1}\right)=Q_{1} \times A_{1} \stackrel{\text { proj }}{\longrightarrow} Q_{1} \stackrel{\alpha}{\rightarrow} Q_{2},
$$

and $\psi_{1}=c_{e} \circ \psi_{2} \circ \alpha$. Similarly, $\left(Q_{2}, \psi_{2}\right) \succeq\left(Q_{1}, \psi_{1}\right)$.

Since only contributors play a role in determining $m(X, B P)$, we can refine the definition of $\operatorname{Split} Q$ as follows: Define

$$
\text { Split }_{\mathrm{T}} Q=\left\{\pi_{j}: P_{j} \rightarrow Q_{j}\right\}
$$

to be conjugacy classes of retractions where $Q_{j} \leqslant P_{j} \leqslant P$ and $Q_{j}$ has the same type as $Q$. As before, we will fix isomorphisms $\psi_{j}: Q_{j} \rightarrow Q$. 
As in Section 4, let

$$
\omega_{i j}=\sum_{N\left(Q_{i}, P_{j}\right) / P_{j}} \psi_{j} \circ \pi_{j} \circ c_{x^{-1}} \circ \psi_{i}^{-1} \in \mathbb{F}_{p} \text { Out } Q .
$$

For the rest of this section, let $n=\left|\operatorname{Split}_{\mathrm{T}} Q\right|$. Define $\mathcal{A}_{T}(Q)=\left(\omega_{i j}\right) \in \operatorname{Mat}_{n}(R)$ and $\mathcal{A}_{T}\left(Q, M^{*}\right)=\left(W_{i j}\right) \in \operatorname{Mat}_{n m}(\mathcal{K})$ as analogues to $\mathcal{A}(Q)$ and $\mathcal{A}\left(Q, M^{*}\right)$ respectively.

Theorem 5.3. $m(X, B P)=\operatorname{rank}_{\mathcal{K}} \mathcal{A}_{T}\left(Q, M^{*}\right)$.

Proof. The proof of Theorem 0.1 in $[\mathbf{9}]$ works in this refined setting.

Among the $n$ retractions in $\operatorname{Split}_{\mathrm{T}} Q$ are the $k$ identity maps $Q_{j} \rightarrow Q_{j}$. Order the retractions in $\operatorname{Split}_{\mathrm{T}} Q$ in such a way that the first $k$ retractions are the $k$ identity maps.

Let $\mathcal{B}(Q)$ be the $k \times n$ submatrix of $\mathcal{A}_{T}(Q)$ obtained by considering only the first $k$ rows of $\mathcal{A}_{T}(Q)$. Define $\mathcal{B}\left(Q, M^{*}\right)$ to be the corresponding $k m \times n m$ submatrix of $\mathcal{A}_{T}\left(Q, M^{*}\right)$. More specifically, we think of $\mathcal{B}(Q)$ as a map $\left(M^{*}\right)^{n} \rightarrow\left(M^{*}\right)^{k}$, and $\mathcal{B}\left(Q, M^{*}\right)$ as a map $\mathcal{K}^{n m} \rightarrow \mathcal{K}^{k m}$.

Proposition 5.4. $\operatorname{rank}_{\mathcal{K}} \mathcal{B}\left(Q, M^{*}\right)=\operatorname{rank}_{\mathcal{K}} \mathcal{A}_{T}\left(Q, M^{*}\right)$.

Proof. Consider row $t$ of the matrix $\mathcal{A}_{T}(Q)$, where $t>k$. We know that $Q_{t}=Q_{i}$ for some $i=1, \ldots, k$. Thus, $\omega_{t j}=\omega_{i j}$ for all $j=1, \ldots, n$. The matrix $\mathcal{A}_{T}\left(Q, M^{*}\right)$ row reduces to a matrix that has $\mathcal{B}\left(Q, M^{*}\right)$ in the top $\mathrm{km} \times \mathrm{nm}$ block, followed by a $(n-k) m \times n m$ block of zeroes. Clearly $\operatorname{rank}_{\mathcal{K}} \mathcal{B}\left(Q, M^{*}\right)=\operatorname{rank}_{\mathcal{K}} \mathcal{A}_{T}\left(Q, M^{*}\right)$.

\subsection{The map from Benson-Feshbach to Martino-Priddy}

The basic idea is to define $\Phi: M \otimes_{R} \bar{L}(P, Q) \rightarrow\left(M^{*}\right)^{n}$ by sending $s \otimes \bar{f}_{Q_{i}, \psi_{i}}$ to the image of $s^{*}$ under the action of row $i$ of $\mathcal{B}(Q)$. We will essentially show that $\operatorname{Ker} \Phi=\mathcal{M}$ and $\operatorname{Im} \Phi=\operatorname{Im} \mathcal{B}\left(Q, M^{*}\right)$. Thus, $\bar{L}(P, Q, M) \cong \operatorname{Im} \mathcal{B}\left(Q, M^{*}\right)$ and the dimension of $\bar{L}(P, Q, M)$ equals the rank of $\mathcal{B}\left(Q, M^{*}\right)$.

Consider elements of $M$ as rows, while elements of $M^{*}$ will be written as columns. Let $\bar{f}_{Q^{\prime}, \psi^{\prime}} \in \bar{L}(P, Q)$ where $Q^{\prime}$ has the same type as $Q$ and $\psi^{\prime}: Q^{\prime} \rightarrow Q$ is an isomorphism. Define

$$
\Phi: M \otimes_{R} \bar{L}(P, Q) \rightarrow\left(M^{*}\right)^{n}
$$

by $\Phi\left(s \otimes \bar{f}_{Q^{\prime}, \psi^{\prime}}\right)=\left[\omega_{\psi^{\prime} 1} \cdot s^{*}, \omega_{\psi^{\prime} 2} \cdot s^{*}, \cdots, \omega_{\psi^{\prime} n} \cdot s^{*}\right]^{T}$ where

$$
\omega_{\psi^{\prime} j}=\sum_{x \in N\left(Q^{\prime}, P_{j}\right) / P_{j}}\left(Q \stackrel{\left(\psi^{\prime}\right)^{-1}}{\rightarrow} Q^{\prime} \stackrel{c_{x-1}}{\rightarrow} P_{j} \stackrel{\pi_{j}}{\rightarrow} Q_{j} \stackrel{\psi_{j}}{\rightarrow} Q\right)
$$

is an element of $\mathbb{F}_{p}$ Out $Q$. (Note: If $\psi^{\prime}=\psi_{i}$ for some $i$, then $\omega_{\psi^{\prime} j}=\omega_{i j}$.) 
Homology, Homotopy and Applications, vol. 8(2), 2006

If $\nu=\sum_{i=1}^{k} s_{i} \otimes \bar{f}_{Q_{i}, \psi_{i}}$ is a typical element of $M \otimes_{R} \bar{L}(P, Q)$, then

$$
\begin{aligned}
\Phi(\nu) & =\sum_{i=1}^{k} \Phi\left(s_{i} \otimes \bar{f}_{Q_{i}, \psi_{i}}\right) \\
& =\left[\begin{array}{l}
\omega_{11} \cdot s_{1}^{*} \\
\omega_{12} \cdot s_{1}^{*} \\
\vdots \\
\omega_{1 n} \cdot s_{1}^{*}
\end{array}\right]+\cdots+\left[\begin{array}{l}
\omega_{k 1} \cdot s_{k}^{*} \\
\omega_{k 2} \cdot s_{k}^{*} \\
\vdots \\
\omega_{k n} \cdot s_{k}^{*}
\end{array}\right] \\
& =\left[\begin{array}{l}
\sum_{i=1}^{k} \omega_{i 1} \cdot s_{i}^{*} \\
\vdots \\
\sum_{i=1}^{k} \omega_{i n} \cdot s_{i}^{*}
\end{array}\right] \\
& =\mathcal{B}(Q)^{T} \cdot\left[\begin{array}{c}
s_{1}^{*} \\
s_{2}^{*} \\
\vdots \\
s_{k}^{*}
\end{array}\right] \in\left(M^{*}\right)^{n}
\end{aligned}
$$

Proposition 5.5. The map $\Phi$ is well-defined.

Proof. Let $\alpha \in \overline{N_{P}\left(Q_{i}\right)}$, where $\alpha=\psi_{i} \circ c_{g} \circ \psi_{i}^{-1}$ for some $g \in N_{P}\left(Q_{i}\right)$. We need $\Phi\left(s \otimes \bar{f}_{Q_{i}, \psi_{i}}\right)=\Phi\left(s \alpha \otimes \bar{f}_{Q_{i}, \psi_{i}}\right)$.

Now $\Phi\left(s \alpha \otimes \bar{f}_{Q_{i}, \psi_{i}}\right)=\Phi\left(s \otimes \bar{f}_{Q_{i}, \alpha \circ \psi_{i}}\right)=\left[\oplus_{j=1}^{n} \omega_{\left(\alpha \circ \psi_{i}\right) j} \cdot s^{*}\right]^{T}$, where

$$
\omega_{\left(\alpha \circ \psi_{i}\right) j}=\sum_{x \in N\left(Q_{i}, P_{j}\right) / P_{j}}\left(Q \stackrel{\left(\alpha \circ \psi_{i}\right)^{-1}}{\longrightarrow} Q_{i} \stackrel{c_{x-1}}{\longrightarrow} P_{j} \stackrel{\pi_{j}}{\rightarrow} Q_{j} \stackrel{\psi_{j}}{\longrightarrow} Q\right) .
$$

Now

$$
\begin{aligned}
\sum_{x \in N\left(Q_{i}, P_{j}\right) / P_{j}} \psi_{j} \circ \pi_{j} \circ c_{x^{-1}} \circ\left(\alpha \circ \psi_{i}\right)^{-1} & =\sum \psi_{j} \circ \pi_{j} \circ c_{x^{-1}} \circ \psi_{i}^{-1} \circ \alpha^{-1} \\
& =\sum \psi_{j} \circ \pi_{j} \circ c_{x^{-1}} \circ \psi_{i}^{-1} \circ \psi_{i} \circ c_{g^{-1}} \circ \psi_{i}^{-1} \\
& =\sum \psi_{j} \circ \pi_{j} \circ c_{x^{-1}} \circ c_{g^{-1}} \circ \psi_{i}^{-1} \\
& =\sum \psi_{j} \circ \pi_{j} \circ c_{x^{-1} g^{-1}} \circ \psi_{i}^{-1} .
\end{aligned}
$$

Since $g \in N_{P}\left(Q_{i}\right)$ and $x \in N\left(Q_{i}, P_{j}\right)$, we know $g x \in N\left(Q_{i}, P_{j}\right)$. Furthermore, the map $x \mapsto g x$ is a bijection of $N_{P}\left(Q_{i}\right)$. Thus

$$
\sum_{x \in N\left(Q_{i}, P_{j}\right)} \psi_{j} \circ \pi_{j} \circ c_{x^{-1} g^{-1}} \circ \psi_{i}^{-1}=\sum_{x \in N\left(Q_{i}, P_{j}\right)} \psi_{j} \circ \pi_{j} \circ c_{x^{-1}} \circ \psi_{i}^{-1} .
$$

We have $\omega_{\left(\alpha \circ \psi_{i}\right) j}=\omega_{i j}$ for all $j=1, \ldots, n$, and so $\Phi\left(s \alpha \otimes \bar{f}_{Q_{i}, \psi_{i}}\right)=\Phi\left(s \otimes \bar{f}_{Q_{i}, \psi_{i}}\right)$.

We need to consider the action of elements of $\bar{A}(P, P)$ on $\bar{L}(P, Q, M)$, especially those corresponding to the retracts. 
Lemma 5.6. $\zeta_{P_{j}, \pi_{j}}\left(s_{i} \otimes \bar{f}_{Q_{i}, \psi_{i}}\right)=s_{i} \cdot \omega_{i j}^{*} \otimes \bar{f}_{Q_{j}, \psi_{j}}$.

Proof. We have

$$
\zeta_{P_{j}, \pi_{j}}\left(s_{i} \otimes \bar{f}_{Q_{i}, \psi_{i}}\right)=\sum_{\substack{x \in Q_{i} \backslash P / P_{j} \\ Q_{i} \leqslant \text { StabP } M \leqslant x_{j} \\ Q_{i} \cap x\left(\operatorname{Ker} \pi_{j}\right)=1}} s_{i} \otimes f_{\pi_{j}\left(x^{-1} Q_{i}\right), \psi_{i} \circ c_{x} \circ \pi_{j}^{-1}} .
$$

The condition $x \in Q_{i} \backslash P / P_{j}$ can be replaced by $x \in P / P_{j}$ ([4], p.34), and the condition $Q_{i} \leqslant \operatorname{Stab}_{P} M \leqslant{ }^{x} P_{j}$ can be replaced by $Q_{i} \leqslant{ }^{x} P_{j}$ ([2], Lemma 4.2). The two replacement conditions are equivalent to the condition $x \in N\left(Q_{i}, P_{j}\right) / P_{j}$.

Now $x^{-1} Q_{i} x \leqslant P_{j}$ and $x^{-1} Q_{i} x \cap \operatorname{Ker} \pi_{j}=1$, so $\pi_{j}$ is one-to-one, hence onto, when restricted to $x^{-1} Q_{i} x$. Thus we have $\pi_{j}\left({ }^{x^{-1}} Q_{i}\right)=Q_{j}$.

Consider a summand of $\omega_{i j}$,

$$
\omega_{i j}(x): Q \stackrel{\psi_{i}^{-1}}{\rightarrow} Q_{i} \stackrel{c_{x-1}}{\rightarrow} x^{-1} Q_{i} x \leqslant P_{j} \stackrel{\pi_{j}}{\rightarrow} Q_{j} \stackrel{\psi_{j}}{\rightarrow} Q,
$$

so $\omega_{i j}=\sum_{x \in N\left(Q_{i}, P_{j}\right) / P_{j}} \omega_{i j}(x)$. Each $\omega_{i j}(x)$ in the sum is an element of Out $Q$. As in the proof of Lemma 5.1, we can write

$$
\omega_{i j}=\sum_{x \in N\left(Q_{i}, P_{j}\right) / P_{j}} \omega_{i j}(x)=\sum_{\substack{x \in N\left(Q_{i}, P_{j}\right) / P_{j} \\ Q_{i} \cap\left(\operatorname{Ker} \pi_{j}\right)=1}} \omega_{i j}(x) .
$$

Each $\omega_{i j}(x)$ is invertible. Let

$$
\omega_{i j}^{-1}(x)=Q \stackrel{\psi_{j}^{-1}}{\rightarrow} Q_{j} \stackrel{\pi_{j}^{-1}}{\rightarrow} x^{-1} Q_{i} x \stackrel{c_{x}}{\rightarrow} Q_{i} \stackrel{\psi_{i}}{\rightarrow} Q .
$$

Then $\omega_{i j}^{*}=\sum_{\substack{x \in N\left(Q_{i}, P_{j}\right) / P_{j} \\ Q_{i} \cap x\left(\operatorname{Ker} \pi_{j}\right)=1}} \omega_{i j}^{-1}(x)$.

Thus, we have

$$
\begin{aligned}
\zeta_{P_{j}, \pi_{j}}\left(s_{i} \otimes \bar{f}_{Q_{i}, \psi_{i}}\right) & =\sum_{\substack{x \in N\left(Q_{i}, P_{j}\right) / P_{j} \\
Q_{i} \cap\left(\operatorname{Ker} \pi_{j}\right)=1}} s_{i} \otimes \bar{f}_{Q_{j}, \psi_{i} \circ c_{x} \circ \pi_{j}^{-1}} \\
& =\sum s_{i} \otimes \bar{f}_{Q_{j}, \psi_{i} \circ c_{x} \circ \pi_{j}^{-1} \circ \psi_{j}^{-1} \circ \psi_{j}} \\
& =\sum s_{i} \otimes \bar{f}_{Q_{j}, \omega_{i j}^{-1}(x) \circ \psi_{j}} \\
& =\sum s_{i} \cdot \omega_{i j}^{-1}(x) \otimes \bar{f}_{Q_{j}, \psi_{j}} \\
& =s_{i} \cdot \omega_{i j}^{*} \otimes \bar{f}_{Q_{j}, \psi_{j}} .
\end{aligned}
$$

Proposition 5.7. $\operatorname{Ker} \Phi=\mathcal{M}$.

Proof. Let $\nu=\sum_{i=1}^{k} s_{i} \otimes \bar{f}_{Q_{i}, \psi_{i}} \in \mathcal{M}=\cap_{\operatorname{Im} \phi \cong Q} \operatorname{Ker} \zeta_{L, \phi}$, where $L \leqslant P$ and $\phi$ : $L \rightarrow P$. 
In particular, for all $j=1, \ldots, n$ we have

$$
\begin{aligned}
0 & =\zeta_{P_{j}, \pi_{j}}(\nu) \\
& =\sum_{i=1}^{k} \zeta_{P_{j}, \pi_{j}}\left(s_{i} \otimes \bar{f}_{Q_{i}, \psi_{i}}\right) \\
& =\sum_{i=1}^{k}\left(s_{i} \cdot \omega_{i j}^{*} \otimes \bar{f}_{Q_{j}, \psi_{j}}\right) \\
& =\left(\sum_{i=1}^{k} s_{i} \cdot \omega_{i j}^{*}\right) \otimes \bar{f}_{Q_{j}, \psi_{j}} .
\end{aligned}
$$

Now by ([5], Lemma 5.21), this implies that for all $j=1, \ldots, n$

$$
\begin{aligned}
0 & =\sum_{i=1}^{k} s_{i} \cdot \omega_{i j}^{*} \\
& =\sum_{i=1}^{k} \omega_{i j} \cdot s_{i}^{*} .
\end{aligned}
$$

Thus we have $\Phi(\nu)=0$ and $\mathcal{M} \subseteq \operatorname{Ker} \Phi$ is proved.

Now let $\nu=\sum_{i=1}^{k} s_{i} \otimes \bar{f}_{Q_{i}, \psi_{i}} \in \operatorname{Ker} \Phi$. If $\nu \notin \mathcal{M}$, then there is a pair $(L, \phi)$, where $\phi: L \rightarrow P$ and $\operatorname{Im} \phi \cong Q$, for which $\nu \notin \operatorname{Ker} \zeta_{L, \phi}$. Consider

$$
\begin{aligned}
\zeta_{L, \phi}(\nu) & =\sum_{i=1}^{k} \zeta_{L, \phi}\left(s_{i} \otimes \bar{f}_{Q_{i}, \psi_{i}}\right) \\
& =\sum_{i=1}^{k}\left[\sum_{\substack{x \in N\left(Q_{i}, L\right) / L \\
Q_{i} \cap \cap^{x}(\operatorname{Ker} \phi)=1}} s_{i} \otimes \bar{f}_{\phi\left(x^{-1} Q_{i}\right), \psi_{i} \circ c_{x} \circ \phi^{-1}}\right] .
\end{aligned}
$$

There must exist at least one pair $\left(Q_{i}, x\right)$ satisfying the conditions in the sum. Let $\left(Q_{i}, x\right)$ be such a pair. Note that $x \in N\left(Q_{i}, L\right)$ implies $x^{-1} Q_{i} x \leqslant L$, and $x^{-1} Q_{i} x \cap$ Ker $\phi=1$ implies $\left.\phi\right|_{x^{-1} Q_{i} x}$ is one-to-one, hence onto $\phi(L)=\bar{Q}$. Let $\bar{\phi}=\left.\phi\right|_{x^{-1} Q_{i} x}$ so $\bar{\phi}: x^{-1} Q_{i} x \rightarrow \bar{Q}$ is an isomorphism. Consider the composition

$$
r_{x}: L \stackrel{\phi}{\rightarrow} \bar{Q} \stackrel{\bar{\phi}^{-1}}{\longrightarrow} x^{-1} Q_{i} x
$$

Now $r_{x}$ is a retraction onto a conjugate of $Q_{i}$, so the pair $\left(L, r_{x}\right)$ is conjugate to a pair $\left(P_{t}, \pi_{t}\right) \in \operatorname{Split}_{\mathrm{T}} Q$ for some $t=1, \ldots, n$. Since $\nu \in \operatorname{Ker} \Phi$, we know that $0=\sum_{i=1}^{k} \omega_{i j} \cdot s_{i}^{*}=\sum_{i=1}^{k} s_{i} \cdot \omega_{i j}^{*}$ for all $j=1, \ldots, n$. Because $\left(P_{t}, \pi_{t}\right)$ is conjugate to $\left(L, r_{x}\right)$, we have $\zeta_{L, r_{x}}(\nu)=\zeta_{P_{t}, \pi_{t}}(\nu)=\left(\sum_{i=1}^{k} s_{i} \cdot \omega_{i j}^{*}\right) \otimes \bar{f}_{Q_{j}, \psi_{j}}=0 \otimes \bar{f}_{Q_{j}, \psi_{j}}$. Since $\operatorname{Im} \phi \cong Q$ and $\operatorname{Ker} \phi=\operatorname{Ker} r_{x}$, we again see from Lemma 5.21 of [5] that $\zeta_{L, \phi}(\nu)=0$. This is a contradiction, so we must have $\nu \in \mathcal{M}$.

This completes the proof that $\operatorname{Ker} \Phi=\mathcal{M}$.

\subsection{Alternative Transformations}

Define a $\mathcal{K}$-linear version of the homomorphism $\Phi$ as follows. Let $\nu=\sum_{i=1}^{k} \vec{s}_{i} \otimes$ $\bar{f}_{Q_{i}, \psi_{i}} \in M \otimes_{R} \bar{L}(P, Q)$, where we now let $\vec{s}_{i}$ represent an $m$-dimensional vector in the $\mathcal{K}$-module $M$. Define $\Psi: M \otimes_{R} \bar{L}(P, Q) \rightarrow \mathcal{K}^{n m}$ by

$$
\Psi(\nu)=\mathcal{B}\left(Q, M^{*}\right)^{T} \cdot\left[\begin{array}{c}
{\overrightarrow{s_{1}}}^{*} \\
{\overrightarrow{s_{2}}}^{*} \\
\vdots \\
{\overrightarrow{s_{k}}}^{*}
\end{array}\right] \in \mathcal{K}^{n m} .
$$

Following the proofs of Propositions 5.5 and 5.7, we see that $\Psi$ is well-defined and $\operatorname{Ker} \Psi=\mathcal{M}$. 
Taking this one step further, let $d=\operatorname{dim}_{\mathbb{F}_{p}} \mathcal{K}$ and let $\overline{\mathcal{B}}\left(Q, M^{*}\right)$ denote the matrix $\mathcal{B}\left(Q, M^{*}\right)$ considered as a $d k m \times d n m$ matrix over $\mathbb{F}_{p}$. Define $\chi: M \otimes_{R} \bar{L}(P, Q) \rightarrow$ $\mathbb{F}_{p}^{d n m}$ as an $\mathbb{F}_{p}$-linear analog to $\Phi$ and $\Psi$. Again, we have that $\chi$ is well-defined and Ker $\chi=\mathcal{M}$. Since $\mathbb{F}_{p}$ is a field, $\operatorname{rank}_{\mathbb{F}_{p}} \overline{\mathcal{B}}\left(Q, M^{*}\right)^{T}=\operatorname{rank}_{\mathbb{F}_{p}} \overline{\mathcal{B}}\left(Q, M^{*}\right)$.

\subsection{Computing Multiplicity}

Again, according to Benson-Feshbach, the multiplicity of $X$ in $B P$, where $X$ originates in $B Q$, is

$$
m_{\mathrm{BF}}(X, B P)=\operatorname{dim}_{\operatorname{End}_{\bar{A}(P, P)} \bar{L}(P, Q, M)} \bar{L}(P, Q, M) .
$$

(The "BF" subscript simply refers to Benson and Feshbach.)

On the other hand, according to Martino-Priddy, the multiplicity of $X$ in $B P$ is

$$
m_{\mathrm{MP}}(X, B P)=\operatorname{rank}_{\mathcal{K}} \mathcal{B}\left(Q, M^{*}\right) .
$$

Catalano proves that when $\bar{L}(P, Q, M) \neq 0, \operatorname{End}_{\bar{A}(P, P)} \bar{L}(P, Q, M) \cong \mathcal{K}$ (see [4], Lemma 3.2).

Finally,

$$
\begin{aligned}
m_{\mathrm{BF}}(X, B P) & =\operatorname{dim}_{\operatorname{End}_{\bar{A}(P, P)} \bar{L}(P, Q, M)} \bar{L}(P, Q, M) \\
& =\operatorname{dim}_{\mathcal{K}} \bar{L}(P, Q, M) \\
& =\left(\frac{1}{d}\right) \operatorname{dim}_{\mathbb{F}_{p}} \bar{L}(P, Q, M) \\
& =\left(\frac{1}{d}\right) \operatorname{dim}_{\mathbb{F}_{p}} \operatorname{Im} \chi \\
& =\left(\frac{1}{d}\right) \operatorname{dim}_{\mathbb{F}_{p}} \operatorname{Im} \overline{\mathcal{B}}\left(Q, M^{*}\right)^{T} \\
& =\left(\frac{1}{d}\right) \operatorname{rank}_{\mathbb{F}_{p}} \overline{\mathcal{B}}\left(Q, M^{*}\right)^{T} \\
& =\left(\frac{1}{d}\right) \operatorname{rank}_{\mathbb{F}_{p}} \mathcal{\mathcal { B }}\left(Q, M^{*}\right) \\
& =\operatorname{rank}_{\mathcal{K}} \mathcal{B}\left(Q, M^{*}\right) \\
& =m_{\mathbb{P P}}(X, B P) .
\end{aligned}
$$

We see, then, a bridge from the work of Benson-Feshbach to the work of MartinoPriddy.

\section{An Example}

Using the notation of Hall and Senior [6], let $P=16 \Gamma_{2} c_{1}=(A \times B) \rtimes C$, where $A=\mathbb{Z}_{4}\langle a\rangle, B=\mathbb{Z}_{2}\langle b\rangle$, and $C=\mathbb{Z}_{2}\langle c\rangle$ with $c a c^{-1}=a b$. Martino and Priddy determined the complete stable splitting of $B P$ in [9].

Let $Q=\mathbb{Z}_{2}\langle c\rangle$. The elements of order 2 in $P$ are $a^{2}, b, c, a^{2} b, a^{2} c, b c, a^{2} b c$. These elements fall into 5 conjugacy classes: $\mathrm{Cl}\left(a^{2}\right)=\left\{a^{2}\right\}, \mathrm{Cl}(b)=\{b\}, \mathrm{Cl}(c)=\{c, b c\}$, $\mathrm{Cl}\left(a^{2} b\right)=\left\{a^{2} b\right\}$, and $\mathrm{Cl}\left(a^{2} c\right)=\left\{a^{2} c, a^{2} b c\right\}$. Take as conjugacy class representatives of groups of the same type as $Q$ the elements $c$ and $a^{2} c$. Let $Q_{1}=\langle c\rangle$ and $Q_{2}=\left\langle a^{2} c\right\rangle$.

\subsection{The Martino-Priddy Matrix}

If $P_{j} \rightarrow Q_{j}$ is a retract, then $\omega_{i j}$ will be zero for all $i$ unless $\operatorname{Stab}_{P} M$ is a subgroup of a conjugate of $P_{j}$. Since Out $Q=1$, there is only one simple $\mathbb{F}_{2}$ Out $Q$-module, namely $M=\mathbb{F}_{2}$ with trivial action. In this case, $\operatorname{Stab}_{P} M=N_{P}\left(Q_{i}\right)$ which equals $\left\langle a^{2}, b, c\right\rangle$ for $i=1,2$. The only subgroups $L$ of $P$ for which $\left\langle a^{2}, b, c\right\rangle$ is a subgroup of a conjugate of $L$ are $L=\left\langle a^{2}, b, c\right\rangle$ and $L=P$. 
There are 4 retracts from $\left\langle a^{2}, b, c\right\rangle$ to each of $Q_{1}$ and $Q_{2}$. For the retracts to $Q_{1}$, the generators $a^{2}, b, c$ map to $e, e, c$ respectively under the first retract $\pi_{1}$, to $e, c$, $c$ under the second $\pi_{2}$, to $c, e, c$ under $\pi_{3}$, and to $c, c, c$ under $\pi_{4}$. For the retracts to $Q_{2}$, the generators $a^{2}, b, c$ map to $e, e, a^{2} c$ under the first retract $\pi_{5}$, to $e, a^{2} c$, $a^{2} c$ under the second $\pi_{6}$, to $a^{2} c, e, e$ under $\pi_{7}$, and to $a^{2} c, a^{2} c, e$ under $\pi_{8}$.

There are two retracts from $P$ to $Q_{1}$ (and two to $Q_{2}$ ). The first retract sends the generators $a, b, c$ to $e, e, c$ respectively (and to $e, e, a^{2} c$ in $Q_{2}$ ), while the second retract maps the generators to $c, e, c$ respectively (and to $a^{2} c, e, a^{2} c$ in $Q_{2}$ ). The retracts to $Q_{1}$ will be denoted $\pi_{9}$ and $\pi_{10}$, while the retracts to $Q_{2}$ are $\pi_{11}$ and $\pi_{12}$.

There are a total of 12 elements in $\operatorname{Split}_{\mathrm{T}} Q$.

Since Out $Q=1$, the entries of the $2 \times 12$ matrix $\mathcal{B}(Q)$ will be sums of 0 's and 1 's. When $P_{j}=\left\langle a^{2}, b, c\right\rangle, \quad N\left(Q_{i}, P_{j}\right) / P_{j}=P / P_{j}=\langle\bar{a}\rangle \cong \mathbb{Z}_{2}$. When $P_{j}=P$, $N\left(Q_{i}, P_{j}\right) / P_{j}=P / P=\bar{e}$. We get

$$
\begin{aligned}
B(Q) & =\left[\begin{array}{lllllllllllll}
1+1 & 1+0 & 1+1 & 1+0 & 1+1 & 1+0 & 0+0 & 0+1 & 1 & 1 & 1 & 1 \\
1+1 & 1+0 & 0+0 & 1+0 & 1+1 & 1+0 & 1+1 & 1+0 & 1 & 1 & 1 & 1
\end{array}\right] \\
& =\left[\begin{array}{llllllllllll}
0 & 1 & 0 & 1 & 0 & 1 & 0 & 1 & 1 & 1 & 1 & 1 \\
0 & 1 & 0 & 1 & 0 & 1 & 0 & 1 & 1 & 1 & 1 & 1
\end{array}\right] .
\end{aligned}
$$

Since $M=\mathbf{F}_{2}$ has dimension 1 and trivial action, $\mathcal{B}(Q)=\mathcal{B}\left(Q, M^{*}\right)$. We see that the matrix has rank 1 , so there is one copy of $B \mathbb{Z}_{2}$ in $B P$.

\subsection{The Benson-Feshbach Modules}

Let $\psi_{1}: Q_{1} \rightarrow Q$ and $\psi_{2}: Q_{2} \rightarrow Q$ be isomorphisms. As above $M=\mathbb{F}_{2}$ is the trivial $\mathbb{F}_{2}$ Out $Q$-module. We know that $\mathbb{F}_{2} \otimes \bar{L}(P, Q)=\left(\mathbb{F}_{2} \otimes \bar{f}_{Q_{1}, \psi_{1}}\right) \oplus\left(\mathbb{F}_{2} \otimes \bar{f}_{Q_{2}, \psi_{2}}\right)$.

When computing $\zeta_{L, \phi}\left(1 \otimes \bar{f}_{Q_{i}, \psi_{i}}\right)$, we need only consider those subgroups $L$ of $P$ for which $\operatorname{Stab}_{P} \mathbb{F}_{2}$ is a subgroup of a conjugate of $L$. As we saw in the previous section, this implies $L=\left\langle a^{2}, b, c\right\rangle$ or $L=P$.

Suppose $\phi: L \rightarrow Q_{i}$ is surjective. If $L=P$, then $\operatorname{Ker} \phi$ is isomorphic to $\mathbb{Z}_{2} \times \mathbb{Z}_{2} \times$ $\mathbb{Z}_{2}$ or $\mathbb{Z}_{4} \times \mathbb{Z}_{2}$

There is only one copy of $\mathbb{Z}_{2} \times \mathbb{Z}_{2} \times \mathbb{Z}_{2}$ in $P$, namely $\left\langle a^{2}, b, c\right\rangle$. In this case, when computing $\zeta_{P, \phi}\left(1 \otimes \bar{f}_{Q_{i}, \psi_{i}}\right)$, the sum runs over all $x \in N\left(Q_{i}, P\right) / P=P / P$ such that $Q_{i} \cap^{x} \operatorname{Ker} \phi=1$. But when $x=e, Q_{i} \cap^{e} \operatorname{Ker} \phi=Q_{i} \cap\left\langle a^{2}, b, c\right\rangle \neq 1$. So $\operatorname{Ker} \zeta_{P, \phi}=M \otimes \bar{L}(P, Q)$.

There are two conjugacy classes of subgroups of $P$ isomorphic to $\mathbb{Z}_{4}$. Choose conjugacy class representatives $L_{1}=\langle a\rangle$ and $L_{2}=\langle a c\rangle$. No matter what $i$ and $j$ are, $L_{i} \times Q_{j}=\langle a, c\rangle$. In this case, when computing $\zeta_{P, \phi}\left(1 \otimes \bar{f}_{Q_{i}, \psi_{i}}\right)$, the sum runs over all $x \in N\left(Q_{i}, P\right) / P=P / P$ such that $Q_{i} \cap^{x} \operatorname{Ker} \phi=1$. But when $x=e, Q_{i} \cap^{e}$ $\operatorname{Ker} \phi=Q_{i} \cap\langle a, c\rangle \neq 1$. So $\operatorname{Ker} \zeta_{P, \phi}=M \otimes \bar{L}(P, Q)$.

Now suppose $L=\left\langle a^{2}, b, c\right\rangle$, then $\operatorname{Ker} \phi$ is isomorphic to $\mathbb{Z}_{2} \times \mathbb{Z}_{2}$. There are 7 subgroups of $P$ isomorphic to $\mathbb{Z}_{2} \times \mathbb{Z}_{2}: K_{1}=\left\langle a^{2}, b\right\rangle, K_{2}=\left\langle a^{2}, c\right\rangle, K_{3}=\left\langle a^{2}, b c\right\rangle$, $K_{4}=\langle b, c\rangle, K_{5}=\left\langle b, a^{2} c\right\rangle, K_{6}=\left\langle a^{2} b, c\right\rangle$, and $K_{7}=\left\langle a^{2} b, a^{2} c\right\rangle$.

No matter what the kernel is, when computing $\zeta_{L, \phi}\left(1 \otimes \bar{f}_{Q_{i}, \psi_{i}}\right)$, the sum runs over all $x \in N\left(Q_{i}, L\right) / L=P / L=\{\bar{e}, \bar{a}\}$ such that $Q_{i} \cap^{x} \operatorname{Ker} \phi=1$. Conjugation by $a$ on the $K_{i}$ yields: ${ }^{a} K_{1}=K_{1},{ }^{a} K_{2}=K_{3},{ }^{a} K_{3}=K_{2},{ }^{a} K_{4}=K_{4},{ }^{a} K_{5}=K_{5},{ }^{a} K_{6}=K_{7}$, and ${ }^{a} K_{7}=K_{6}$. 
Suppose the image of $\phi$ is $Q_{j}$, then we get the following intersections and actions:

$$
\begin{aligned}
& Q_{1} \cap K_{1}=1, \quad Q_{1} \cap{ }^{a} K_{1}=1 \quad \Rightarrow \zeta_{L, \phi}\left(1 \otimes \bar{f}_{Q_{1}, \psi_{1}}\right)=2\left(1 \otimes \bar{f}_{Q_{j}, \psi_{j}}\right)=0 \\
& Q_{1} \cap K_{2} \neq 1, \quad Q_{1} \cap{ }^{a} K_{2}=1 \Rightarrow \bar{f}_{L, \phi}\left(1 \otimes \bar{f}_{Q_{1}, \psi_{1}}\right)=1 \otimes \bar{f}_{Q_{j}, \psi_{j}} \\
& Q_{1} \cap K_{3}=1, \quad Q_{1} \cap{ }^{a} K_{3} \neq 1 \quad \Rightarrow \zeta_{L, \phi}\left(1 \otimes \bar{f}_{Q_{1}, \psi_{1}}\right)=1 \otimes \bar{f}_{Q_{j}, \psi_{j}} \\
& Q_{1} \cap K_{4} \neq 1, \quad Q_{1} \cap{ }^{a} K_{4} \neq 1 \Rightarrow \zeta_{L, \phi}\left(1 \otimes \bar{f}_{Q_{1}, \psi_{1}}\right)=0 \\
& Q_{1} \cap K_{5}=1, \quad Q_{1} \cap{ }^{a} K_{5}=1 \Rightarrow \zeta_{L, \phi}\left(1 \otimes \bar{f}_{Q_{1}, \psi_{1}}\right)=2\left(1 \otimes \bar{f}_{Q_{j}, \psi_{j}}\right)=0 \\
& Q_{1} \cap K_{6} \neq 1, \quad Q_{1} \cap{ }^{a} K_{6}=1 \Rightarrow \zeta_{L, \phi}\left(1 \otimes \bar{f}_{Q_{1}, \psi_{1}}\right)=1 \otimes \bar{f}_{Q_{j}, \psi_{j}} \\
& Q_{1} \cap K_{7}=1, \quad Q_{1} \cap{ }^{a} K_{7} \neq 1 \Rightarrow \zeta_{L, \phi}\left(1 \otimes \bar{f}_{Q_{1}, \psi_{1}}\right)=1 \otimes \bar{f}_{Q_{j}, \psi_{j}} \\
&
\end{aligned}
$$

We see that $\operatorname{Ker} \zeta_{L, \phi}$ is either all of $M \otimes \bar{L}(P, Q)$, or just the submodule generated by $1 \otimes \bar{f}_{Q_{1}, \psi_{1}}+1 \otimes \bar{f}_{Q_{2}, \psi_{2}}$. Thus $\mathcal{M}=\left\langle 1 \otimes \bar{f}_{Q_{1}, \psi_{1}}+1 \otimes \bar{f}_{Q_{2}, \psi_{2}}\right\rangle$, and $\bar{L}(P, Q, M)$ is one-dimensional.

\subsection{The map $\Phi$}

We will make a couple of computations to show how the map $\Phi$ works, and to confirm that $\mathcal{M}=\operatorname{Ker} \Phi$.

Consider $\nu=1 \otimes \bar{f}_{Q_{1}, \psi_{1}}+1 \otimes \bar{f}_{Q_{2}, \psi_{2}} \in \mathcal{M}$.

$$
\Phi(\nu)=\left[\begin{array}{cc}
0 & 0 \\
1 & 1 \\
0 & 0 \\
1 & 1 \\
0 & 0 \\
1 & 1 \\
0 & 0 \\
1 & 1 \\
1 & 1 \\
1 & 1 \\
1 & 1 \\
1 & 1
\end{array}\right] \cdot\left[\begin{array}{c}
1^{*} \\
1^{*}
\end{array}\right]=\left[\begin{array}{l}
0 \\
0 \\
0 \\
0 \\
0 \\
0 \\
0 \\
0 \\
0 \\
0 \\
0 \\
0
\end{array}\right]
$$

So we see that $\mathcal{M} \subseteq \operatorname{Ker} \Phi$. 
Now let $\nu=\sum_{i=1}^{2} s_{i} \otimes \bar{f}_{Q_{i}, \psi_{i}} \in \operatorname{Ker} \Phi$. Then

$$
\Phi(\nu)=\left[\begin{array}{cc}
0 & 0 \\
1 & 1 \\
0 & 0 \\
1 & 1 \\
0 & 0 \\
1 & 1 \\
0 & 0 \\
1 & 1 \\
1 & 1 \\
1 & 1 \\
1 & 1 \\
1 & 1
\end{array}\right] \cdot\left[\begin{array}{c}
s_{1}^{*} \\
s_{2}^{*}
\end{array}\right]=\left[\begin{array}{c}
0 \\
s_{1}^{*}+s_{2}^{*} \\
0 \\
s_{1}^{*}+s_{2}^{*} \\
0 \\
s_{1}^{*}+s_{2}^{*} \\
0 \\
s_{1}^{*}+s_{2}^{*} \\
s_{1}^{*}+s_{2}^{*} \\
s_{1}^{*}+s_{2}^{*} \\
s_{1}^{*}+s_{2}^{*} \\
s_{1}^{*}+s_{2}^{*}
\end{array}\right]=\overrightarrow{0 .}
$$

We see that either $s_{1}=s_{2}=0$ or $s_{1}=s_{2}=1$.

Assume $s_{1}=s_{2}=1$, and consider $\zeta_{L, \phi}(\nu)$ where $L=\left\langle a^{2}, b, c\right\rangle$ and $\phi: L \rightarrow Q_{2}$ sends each of the generators $a^{2}, b$, and $c$ to $a^{2} c$ in $Q_{2}$. In particular, note that $\phi$ is not a retract.

Now

$$
\begin{aligned}
& \zeta_{L, \phi}(\nu)=\zeta_{L, \phi}\left(1 \otimes \bar{f}_{Q_{1}, \psi_{1}}\right)+\zeta_{L, \phi}\left(1 \otimes \bar{f}_{Q_{2}, \psi_{2}}\right) \\
& =\sum 1 \otimes \bar{f}_{\phi\left(x^{-1} Q_{1}\right), \psi_{1} \circ c_{x} \circ \phi^{-1}}+\sum 1 \otimes \bar{f}_{\phi\left(x^{-1} Q_{2}\right), \psi_{2} \circ c_{x} \circ \phi^{-1}}
\end{aligned}
$$

where the first sum runs over $x \in N\left(Q_{1}, L\right) / L$ with $Q_{1} \cap^{x}(\operatorname{Ker} \phi)=1$ and the second sum runs over $x \in N\left(Q_{2}, L\right) / L$ with $Q_{2} \cap^{x}(\operatorname{Ker} \phi)=1$

As we saw in Section 6.2 , for $i=1,2$ we have $N\left(Q_{i}, L\right) / L=P / L=\mathbb{Z}_{2}\langle\bar{a}\rangle$. Since $\operatorname{Ker} \phi=K_{7}$, we know that $Q_{1} \cap{ }^{e}(\operatorname{Ker} \phi)=1$ and $Q_{1} \cap{ }^{a}(\operatorname{Ker} \phi) \neq 1$. Also, $\phi\left(e^{-1} Q_{1}\right)=Q_{2}$ and $\psi_{1} \circ c_{e} \circ \phi^{-1}=\psi_{2}$. Thus, $\zeta_{L, \phi}\left(1 \otimes \bar{f}_{Q_{1}, \psi_{1}}\right)=1 \otimes \bar{f}_{Q_{2}, \psi_{2}}$. On the other hand, $Q_{2} \cap{ }^{e}(\operatorname{Ker} \phi) \neq 1$, while $Q_{2} \cap{ }^{a}(\operatorname{Ker} \phi)=1$. Also, $\phi\left(\left(^{a^{-1}} Q_{2}\right)=Q_{2}\right.$ and $\psi_{2} \circ c_{a} \circ \phi^{-1}=\psi_{2}$. Thus, $\zeta_{L, \phi}\left(1 \otimes \bar{f}_{Q_{2}, \psi_{2}}\right)=1 \otimes \bar{f}_{Q_{2}, \psi_{2}}$. We conclude that $1 \otimes \bar{f}_{Q_{1}, \psi_{1}}+1 \otimes \bar{f}_{Q_{2}, \psi_{2}} \in \operatorname{Ker} \zeta_{L, \phi}$.

Now $\phi: L \rightarrow Q_{2}$ is not a retract, but $r_{a}=\bar{\phi}^{-1} \circ \phi$ (as defined in the proof of Proposition 5.7) is a retract to $a^{-1} Q_{2} a$. In that proof we argued that $\zeta_{L, \phi}$ acts like $\zeta_{P_{j}, \pi_{j}}$ for some $j$. It is easy to confirm that the pair $\left(L, r_{a}\right)$ is conjugate to the pair $\left(P_{8}, \pi_{8}\right)$. Moreover, one can see from the work above that $\zeta_{P_{8}, \pi_{8}}(\nu)=2(1 \otimes$ $\left.\bar{f}_{Q_{2}, \psi_{2}}\right)=0$.

\section{Acknowledgements}

A special "thank you" goes out to each of Dave, Mark, John and Stewart. Their support encouraged the undertaking of this project and helped secure local funding for it. Also, it is clear that without their fundamental contributions to the subject of stable splittings this paper would be pointless. Finally, thanks to the referee for a careful reading of the paper and several helpful clarifications. 
Homology, Homotopy and Applications, vol. 8(2), 2006

\section{References}

[1] Benson, D. 1996, 'Stably splitting BG', Bull. Amer. Math. Soc., vol. 33, pp. 189-198.

[2] Benson, D. \& Feshbach,M. 1992, 'Stable splittings of classifying spaces of finite groups', Topology, vol. 31, pp. 157-176.

[3] Carlsson, G. 1984, 'Equivariant Stable Homotopy and Segal's Burnside Ring Conjecture,' Ann. of Math., vol. 120, pp. 189-224.

[4] Catalano, M. 1996, 'Stable splittings of BP for some $P$ of order 32', Jour. Pure and App. Alg.' vol. 111, pp. 31-50.

[5] Catalano, M. 1991, 'The stable splitting of $B P$ for some groups $P$ of order 32', Ph.D. Dissertation, University of Minnesota.

[6] Hall, M. \& Senior, J. 1964, The groups of order $2^{n}(n<6)$, MacMillan Co., New York.

[7] Harris, J. 1993, '93d:55013,' in Mathematical Reviews: MR1153243, American Mathematical Society.

[8] Lewis, G., May, P., \& McClure, J. 1982, 'Classifying $G$-spaces and the Segal Conjecture,' CMS Conference Proceedings 2: Current Trends in Algebraic Topology, Part 2, American Mathematical Society, Providence, pp. 165-179.

[9] Martino, J. \& Priddy, S. 1992, 'The complete stable splitting for the classifying space of a finite group,' Topology, vol. 31, pp. 143-156.

[10] Nishida, G. 1985, 'Stable Homotopy Type of Classifying Spaces of Finite Groups,' Algebraic and Topological Theories, Kinokuniya, Tokyo, pp. 391404.

[11] Priddy, S. 1990, 'On characterizing summands in the classifying space of a group, II,' in Lecture Notes in Mathematics 1418: Homotopy theory and related topics (Kinosaki), Springer, Berlin.

Jill Dietz dietz@stolaf.edu

Department of Mathematics, Statistics, and Computer Science

St. Olaf College

1520 St. Olaf Ave.

Northfield, MN 55057

USA

This article is available at http://intlpress.com/HHA/v8/n2/a4 